https://doi.org/10.15407/ujpe64.8.695

P. LEBIEDOWICZ, ${ }^{1}$ A. SZCZUREK, ${ }^{1}$ O. NACHTMANN ${ }^{2}$

${ }^{1}$ Institute of Nuclear Physics, Polish Academy of Sciences

(Radzikowskiego 152, PL-31342 Cracow, Poland; e-mail: Piotr.Lebiedowicz@ifj.edu.pl)

2 Institut fur Theoretische Physik, Universitat Heidelberg

(Philosophenweg 16, D-69120 Heidelberg, Germany)

\title{
SEARCHING FOR ODDERON IN EXCLUSIVE REACTIONS
}

\begin{abstract}
We discuss the possibility to use the $p p \rightarrow p p \phi \phi$ process in identifying the odderon exchange. So far, there is no unambiguous experimental evidence for the odderon, the charge conjugation $C=-1$ counterpart of the $C=+1$ pomeron. Last year, the results of the TOTEM collaboration suggest that the odderon exchange can be responsible for a disagreement of theoretical calculations and the TOTEM data for the elastic proton-proton scattering. Here, we present recent studies for the central exclusive production (CEP) of $\phi \phi$ pairs in proton-proton collisions. We consider the pomeron-pomeron fusion to $\phi \phi(\mathbb{P P} \rightarrow \phi \phi)$ through the continuum processes, due to the $\hat{t}$ - and $\hat{u}$-channel reggeized $\phi$-meson, photon, and odderon exchanges, as well as through the $s$-channel resonance process $\left(\mathbb{P P} \rightarrow f_{2}(2340) \rightarrow \phi \phi\right)$. This $f_{2}$ state is a candidate for a tensor glueball. The amplitudes for the processes are formulated within the tensor-pomeron and vector-odderon approach. Some model parameters are determined from the comparison to the WA102 experimental data. The odderon exchange is not excluded by the WA102 data for high $\phi \phi$ invariant masses. The measurement of large $M_{\phi \phi}$ or $Y_{\text {diff }}$ events at the LHC would therefore suggest the presence of the odderon exchange. The process is advantageous, as here the odderon does not couple to protons.

Keywords: exclusive reactions, meson, Regge physics, pomeron, odderon, LHC.
\end{abstract}

\section{Introduction}

Diffractive studies are one of the important parts of the physics program for the RHIC and LHC experiments. A particularly interesting class is the centralexclusive-production (CEP) processes, where all centrally produced particles are detected.

In recent years, there has been a renewed interest in the exclusive production of $\pi^{+} \pi^{-}$pairs at high energies related to successful experiments by the CDF [1] and the CMS [2] collaborations. These measurements are important in the context of the resonance production, in particular, in searches for glueballs. In the CDF and CMS experiments, the large rapidity gaps around the centrally produced dimeson system were checked, but the forward- and backward-going (anti)protons were not detected. Preliminary results of similar CEP studies have been presented by the ALICE and LHCb collaborations at the LHC. Although such results will have a diffractive nature, fur-

(C) P. LEBIEDOWICZ, A. SZCZUREK,

O. NACHTMANN, 2019

ISSN 2071-0194. Ukr. J. Phys. 2019. Vol. 64, No. 8 ther efforts are needed to ensure their exclusivity. Ongoing and planned experiments at the RHIC (see, e.g., [3]) and future experiments at the LHC will be able to detect all particles produced in central exclusive processes, including the forward- and backward-going protons. The feasibility studies for the $p p \rightarrow p p \pi^{+} \pi^{-}$ process with the tagging of scattered protons, as carried out for the ATLAS and ALFA detectors, are in [4]. Similar possibilities exist using the CMS and TOTEM detectors.

In [21], the tensor-pomeron and vector-odderon concepts were introduced for soft reactions. In this approach, the $C=+1$ pomeron and the reggeons $\mathbb{R}_{+}=f_{2 \mathbb{R}}, a_{2 \mathbb{R}}$ are treated as effective rank- 2 symmetric tensor exchanges, while the $C=-1$ odderon and the reggeons $\mathbb{R}_{-}=\omega_{\mathbb{R}}, \rho_{\mathbb{R}}$ are treated as effective vector exchanges. For these effective exchanges, a number of propagators and vertices, respecting the standard rules of quantum field theory, were derived from comparisons with experiments. This allows for an easy construction of amplitudes for specific processes. In [22], the helicity structure of a 


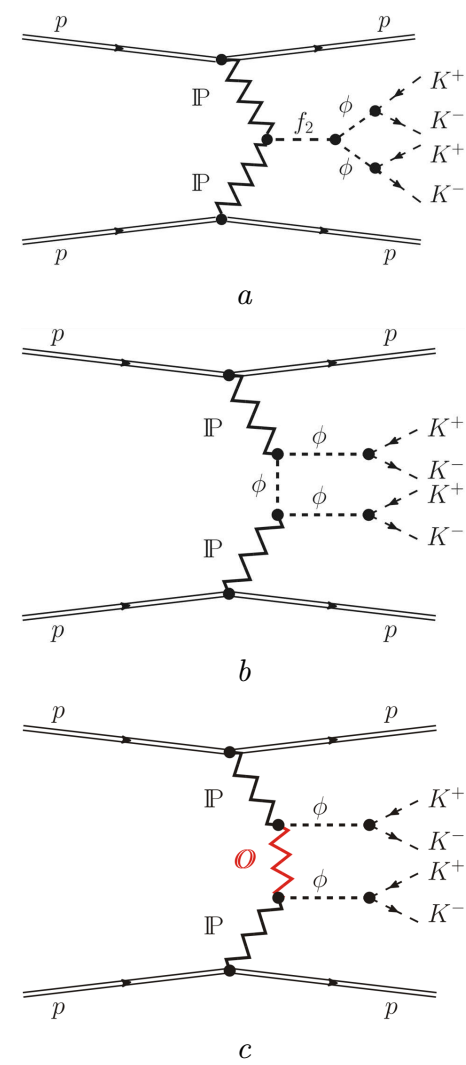

Fig. 1. Born-level diagrams for the double pomeron central exclusive $\phi \phi$ production and their decays into $K^{+} K^{-} K^{+} K^{-}$: $\phi \phi$ production via an $f_{2}$ resonance $(a)$. Other resonances, e.g., of $f_{0^{-}}$and $\eta$-type, can also contribute here. The continuum $\phi \phi$ production via an intermediate $\phi$ and odderon $(\mathbb{O})$ exchanges, respectively, $(b)$ and $(c)$. $\mathbb{P}-\gamma-\mathbb{P}$ and $\mathbb{O}-\mathbb{P}-\mathbb{O}$ contributions are also possible, but negligibly small

small- $|t|$ proton-proton elastic scattering was considered in three models for the pomeron: tensor, vector, and scalar ones. Only the tensor ansatz for the pomeron was found to be compatible with the highenergy experiment on the polarized $p p$ elastic scattering $[10]$.

Applications of the tensor-pomeron and vectorodderon ans'atze were given for the photoproduction of pion pairs in [11] and for a number of centralexclusive-production (CEP) reactions in $p p$ collisions in $[12-20]$. In addition, contributions from the subleading exchanges, $\mathbb{R}_{+}$and $\mathbb{R}_{-}$, were discussed in these works. As an example, for the $p p \rightarrow p p p \bar{p}$ reaction [17], the contributions involving an odderon are expected to be small since its coupling to a proton is very small. We have predicted asymmetries in the (pseudo)rapidity distributions of the centrally produced antiproton and proton. The asymmetry is caused by interference effects of the dominant $(\mathbb{P}, \mathbb{P})$ with the subdominant $\left(\mathbb{O}+\mathbb{R}_{-}, \mathbb{P}+\mathbb{R}_{+}\right)$and $\left(\mathbb{P}+\mathbb{R}_{+}\right.$, $\mathbb{O}+\mathbb{R}_{-}$) exchanges. We find only very small effects for the odderon, roughly a factor of 10 smaller than the effects due to reggeons.

So far, there is no unambiguous experimental evidence of the odderon, the charge conjugation $C=-1$ counterpart of the $C=+1$ pomeron, introduced on theoretical grounds in [5]. A hint of the odderon was seen in ISR results [6] as a small difference between the differential cross-sections of elastic proton-proton $(p p)$ and proton-antiproton $(p \bar{p})$ scatterings in the diffractive dip region at $\sqrt{s}=53 \mathrm{GeV}$. Recently, the TOTEM Collaboration has published data from highenergy elastic $p p$ scattering experiments at the LHC. In [7], results were given for the $\rho$ parameter, the ratio of the real part to the imaginary one of the forward scattering amplitude. The interpretation of these results is controversial at the moment.

As was discussed in [8], the exclusive diffractive $J / \psi$ and $\phi$ productions from the pomeron-odderon fusion in high-energy $p p$ and $p \bar{p}$ collisions are a direct probe for a possible odderon exchange. For a nice review of the odderon physics, see [9]. In the diffractive production of $\phi$ meson pairs, it is possible to have the pomeron-pomeron fusion with intermediate $\hat{t} / \hat{u}$ channel odderon exchange [20]; see the corresponding diagram in Fig. 1, $c$. Thus, the $p p \rightarrow p p \phi \phi$ reaction is a good candidate for the odderon-exchange searches, as it does not involve the coupling of the odderon to the proton.

Studies of different decay channels in the central exclusive production would be very valuable also in the context of identification of glueballs. One of the promising reactions is $p p \rightarrow p p \phi \phi$ with both $\phi \equiv \phi(1020)$ mesons decaying into the $K^{+} K^{-}$channel. Structures in the $\phi \phi$ invariant-mass spectrum were observed by several experiments, e.g., in the exclusive $\pi^{-} p \rightarrow \phi \phi n$ [23] and $K^{-} p \rightarrow \phi \phi \Lambda$ [24] reactions, and in the central production [25]. Three tensor states, $f_{2}(2010), f_{2}(2300)$, and $f_{2}(2340)$, observed previously in [23], were also observed in the radiative decay $J / \psi \rightarrow \gamma \phi \phi[26]$. The nature of these resonances is not understood at present and a tensor glueball has still not been clearly identified. According to lattice-QCD simulations, the lightest tensor glueball has a mass between 2.2 and $2.4 \mathrm{GeV}$, see, 
e.g. [27]. The $f_{2}(2300)$ and $f_{2}(2340)$ states are good candidates to be tensor glueballs.

For an interesting approach to the exclusive diffractive resonance production in $p p$ collisions at high energies, see also Ref. [28, 29].

\section{A Sketch of Formalism}

In [20], we considered the CEP of four charged kaons via the intermediate $\phi \phi$ state. Explicit expressions for the $p p \rightarrow p p \phi \phi$ amplitudes involving the pomeronpomeron fusion to $\phi \phi(\mathbb{P P} \rightarrow \phi \phi)$ through the continuum processes, due to the $\hat{t}$ - and $\hat{u}$-channel reggeized $\phi$-meson, photon, and odderon exchanges, as well as through the $s$-channel resonance reaction $(\mathbb{P P} \rightarrow$ $\left.\rightarrow f_{2}(2340) \rightarrow \phi \phi\right)$ were given there. Here, we discuss briefly the continuum processes for the $p p \rightarrow p p \phi \phi$ reaction.

The "Born-level" amplitude for the $p p \rightarrow p p \phi \phi$ reaction is

$\mathcal{M}^{\text {Born }}=\mathcal{M}^{\left(f_{2}-\text { exchange }\right)}+\mathcal{M}^{(\phi-\text { exchange })}+$

$+\mathcal{M}^{(\mathbb{O}-\text { exchange })}$.

For the continuum process with the odderon exchange (Fig. 1,c), the amplitude is a sum of $\hat{t}$ - and $\hat{u}$ channel amplitudes. The $\hat{t}$-channel term can be written as

$$
\begin{aligned}
& \mathcal{M}^{(\hat{t})}=(-i) \bar{u}\left(p_{1}, \lambda_{1}\right) i \Gamma_{\mu_{1} \nu_{1}}^{(\mathbb{P} p p)}\left(p_{1}, p_{a}\right) u\left(p_{a}, \lambda_{a}\right) \times \\
& \times i \Delta^{(\mathbb{P}) \mu_{1} \nu_{1}, \alpha_{1} \beta_{1}}\left(s_{13}, t_{1}\right) \times \\
& \times i \Gamma_{\rho_{1} \rho_{3} \alpha_{1} \beta_{1}}^{(\mathbb{P O} \phi)}\left(\hat{p}_{t},-p_{3}\right)\left(\epsilon^{(\phi) \rho_{3}}\left(\lambda_{3}\right)\right)^{*} \times \\
& \times i \Delta^{(\mathbb{O}) \rho_{1} \rho_{2}}\left(s_{34}, \hat{p}_{t}\right) \times \\
& \times i \Gamma_{\rho_{4} \rho_{2} \alpha_{2} \beta_{2}}^{(\mathbb{P O} \phi)}\left(p_{4}, \hat{p}_{t}\right)\left(\epsilon^{(\phi) \rho_{4}}\left(\lambda_{4}\right)\right)^{*} \times \\
& \times i \Delta^{(\mathbb{P}) \alpha_{2} \beta_{2}, \mu_{2} \nu_{2}}\left(s_{24}, t_{2}\right) \times \\
& \times \bar{u}\left(p_{2}, \lambda_{2}\right) i \Gamma_{\mu_{2} \nu_{2}}^{(\mathbb{P} p p)}\left(p_{2}, p_{b}\right) u\left(p_{b}, \lambda_{b}\right),
\end{aligned}
$$

where $p_{a, b}, p_{1,2}$ and $\lambda_{a, b}, \lambda_{1,2}= \pm \frac{1}{2}$ denote the fourmomenta and helicities of the protons and $p_{3,4}$ and $\lambda_{3,4}=0, \pm 1$ denote the four-momenta and helicities of the $\phi$ mesons, respectively. $\hat{p}_{t}=p_{a}-p_{1}-p_{3}$, $\hat{p}_{u}=p_{4}-p_{a}+p_{1}, s_{i j}=\left(p_{i}+p_{j}\right)^{2}, t_{1}=\left(p_{1}-p_{a}\right)^{2}$, $t_{2}=\left(p_{2}-p_{b}\right)^{2} . \Gamma^{(\mathbb{P} p p)}$ and $\Delta^{(\mathbb{P})}$ denote the proton ISSN 2071-0194. Ukr. J. Phys. 2019. Vol. 64, No. 8 vertex function and the effective propagator, respectively, for the tensorial pomeron. The corresponding expressions are as follows [21]:

$i \Gamma_{\mu \nu}^{(\mathbb{P} p p)}\left(p^{\prime}, p\right)=-i 3 \beta_{\mathbb{P} N N} F_{1}(t) \times$

$\times\left\{\frac{1}{2}\left[\gamma_{\mu}\left(p^{\prime}+p\right)_{\nu}+\gamma_{\nu}\left(p^{\prime}+p\right)_{\mu}\right]-\frac{1}{4} g_{\mu \nu}\left(\not p^{\prime}+\not p\right)\right\}$,

$i \Delta_{\mu \nu, \kappa \lambda}^{(\mathbb{P})}(s, t)=\frac{1}{4 s}\left(g_{\mu \kappa} g_{\nu \lambda}+g_{\mu \lambda} g_{\nu \kappa}-\frac{1}{2} g_{\mu \nu} g_{\kappa \lambda}\right) \times$

$\times\left(-i s \alpha_{\mathbb{P}}^{\prime}\right)^{\alpha_{\mathbb{P}}(t)-1}$,

where $\beta_{\mathbb{P} N N}=1.87 \mathrm{GeV}^{-1}$. The pomeron trajectory $\alpha_{\mathbb{P}}(t)$ is assumed to be of the standard linear form (see, e.g., [30]): $\alpha_{\mathbb{P}}(t)=\alpha_{\mathbb{P}}(0)+\alpha_{\mathbb{P}}^{\prime} t, \alpha_{\mathbb{P}}(0)=1.0808$, $\alpha_{\mathbb{P}}^{\prime}=0.25 \mathrm{GeV}^{-2}$.

Our ansatz for the effective propagator of the $C=$ $=-1$ odderon is [21]

$i \Delta_{\mu \nu}^{(\mathbb{O})}(s, t)=-i g_{\mu \nu} \frac{\eta_{\mathbb{O}}}{M_{0}^{2}}\left(-i s \alpha_{\mathbb{O}}^{\prime}\right)^{\alpha_{\mathbb{O}}(t)-1}$

with

$M_{0}=1 \mathrm{GeV}, \quad \eta_{\mathbb{D}}= \pm 1$.

Here, $\alpha_{\mathbb{O}}(t)=\alpha_{\mathbb{O}}(0)+\alpha_{\mathbb{O}}^{\prime} t$ and we choose, as an example, $\alpha_{\mathbb{O}}^{\prime}=0.25 \mathrm{GeV}^{-2}, \alpha_{\mathbb{O}}(0)=1.05$.

For the $\mathbb{P O} \phi$ vertex, we use an ansatz with two rank-four tensor functions [20]:

$i \Gamma_{\mu \nu \kappa \lambda}^{(\mathbb{P O} \phi)}\left(k^{\prime}, k\right)=i F^{(\mathbb{P O O} \phi)}\left(\left(k+k^{\prime}\right)^{2}, k^{\prime 2}, k^{2}\right) \times$

$\times\left[2 a_{\mathbb{P O} \phi} \Gamma_{\mu \nu \kappa \lambda}^{(0)}\left(k^{\prime}, k\right)-b_{\mathbb{P O} \phi} \Gamma_{\mu \nu \kappa \lambda}^{(2)}\left(k^{\prime}, k\right)\right]$.

We take the factorized form for the $\mathbb{P} O \phi$ form factor:

$F^{(\mathbb{P O} \phi)}\left(\left(k+k^{\prime}\right)^{2}, k^{\prime 2}, k^{2}\right)=$

$=F\left(\left(k+k^{\prime}\right)^{2}\right) F\left(k^{\prime 2}\right) F^{(\mathbb{P O} \phi)}\left(k^{2}\right)$,

where $F\left(k^{2}\right)=\left(1-k^{2} / \Lambda^{2}\right)^{-1}$ and $F^{(\mathbb{P O} \phi)}\left(m_{\phi}^{2}\right)=1$. The coupling parameters $a_{\mathbb{P O} \phi}, b_{\mathbb{P O} \phi}$ and the cutoff parameter $\Lambda^{2}$ could be adjusted to the WA102 experimental data [25].

At low $\sqrt{s_{34}}=\mathrm{M}_{\phi \phi}$, the Regge type of interaction is not realistic and should be switched off. To achieve this, we multiplied the $\mathbb{O}$-exchange amplitude by a purely phenomenological factor: $F_{\mathrm{thr}}\left(s_{34}\right)=1-$ $\left.-\exp \left[\left(s_{\mathrm{thr}}-s_{34}\right) / s_{\mathrm{thr}}\right)\right]$ with $s_{\mathrm{thr}}=4 m_{\phi}^{2}$. 


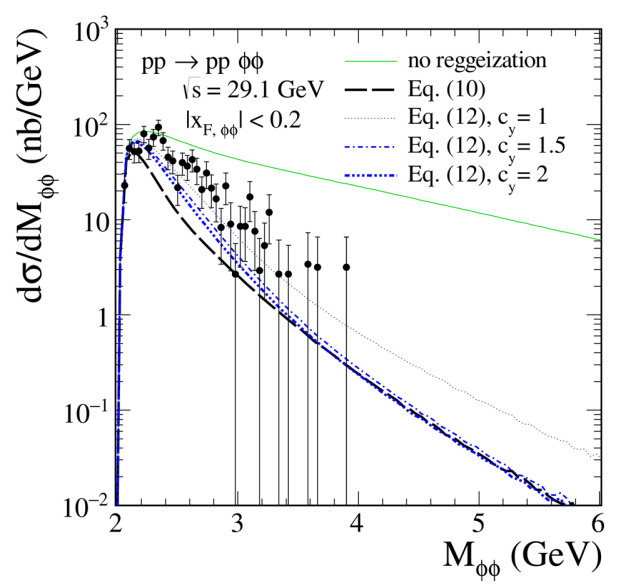

$a$

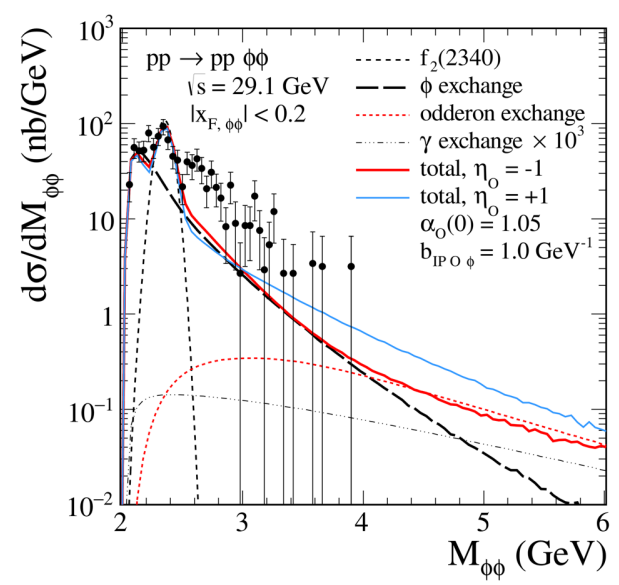

$b$

Fig. 2. Distributions in the $\phi \phi$ invariant mass. The calculations were done for $\sqrt{s}=29.1 \mathrm{GeV}$ and $\left|x_{F, \phi \phi}\right| \leqslant 0.2$. The WA102 experimental data from [25] are shown. In the top panel, the green solid line corresponds to the non-reggeized $\phi$ exchange contribution. The results for two prescriptions of the reggeization, (10) and (12), are shown by the black and blue lines, respectively. In the bottom panel, we show the complete results including the $f_{2}(2340)$-resonance contribution and the continuum processes due to the reggeized- $\phi$, odderon, and photon exchanges. The black long-dashed line corresponds to the $\phi$-exchange contribution and the black dashed line corresponds to the $f_{2}(2340)$ contribution. The red dotted line represents the odderon-exchange contribution for $a_{\mathbb{P O} \phi}=0$ and $b_{\mathbb{P O} \phi}=1.0 \mathrm{GeV}^{-1}$ in (6)

The amplitude for the process shown in Fig. 1, $b$ has the same form as the amplitude with the $\mathbb{O}$ exchange, but we have to make the following replacements:

$i \Gamma_{\mu \nu \kappa \lambda}^{(\mathbb{P O} \phi)}\left(k^{\prime}, k\right) \rightarrow i \Gamma_{\mu \nu \kappa \lambda}^{(\mathbb{P} \phi \phi)}\left(k^{\prime}, k\right)$,

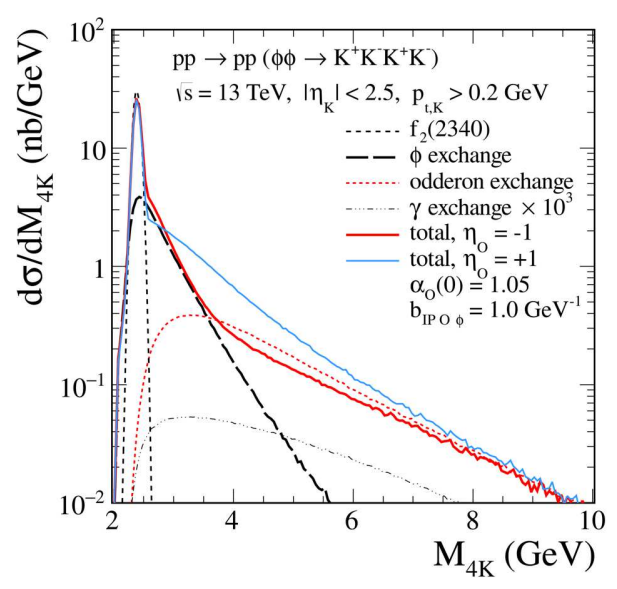

$a$

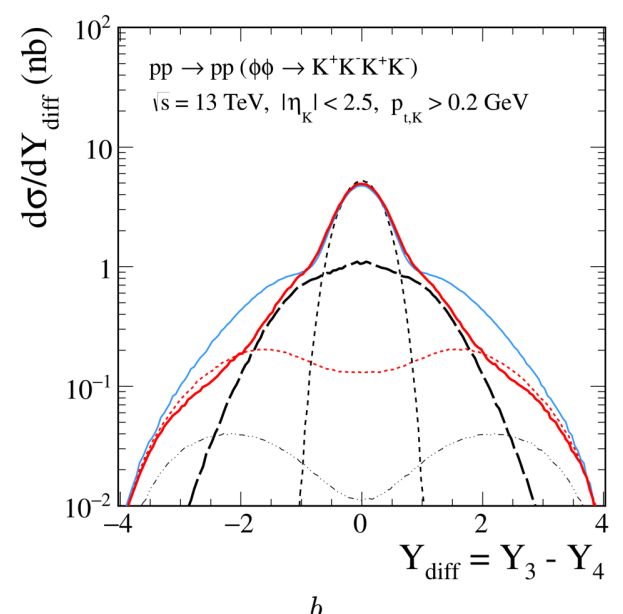

Fig. 3. Distributions in $\mathrm{M}_{4 K}$ (left) and in $\mathrm{Y}_{\text {diff }}$ (right) for the $p p \rightarrow p p\left(\phi \phi \rightarrow K^{+} K^{-} K^{+} K^{-}\right)$reaction calculated for $\sqrt{s}=$ $=13 \mathrm{TeV}$ and $\left|\eta_{K}\right|<2.5, p_{t, K}>0.2 \mathrm{GeV}$. The coherent sum of all terms is shown by the red and blue solid lines for $\eta_{\mathbb{O}}=-1$ and $\eta_{\mathbb{O}}=+1$, respectively. Here, we take $\alpha_{\mathbb{O}}(0)=1.05$. The absorption effects are included in the calculations

$i \Delta_{\mu \nu}^{(\mathbb{O})}\left(s_{34}, \hat{p}^{2}\right) \rightarrow i \Delta_{\mu \nu}^{(\phi)}(\hat{p})$

We have fixed the coupling parameters of the tensor pomeron to the $\phi$ meson, based on the HERA experimental data for the $\gamma p \rightarrow \phi p$ reaction; see [18].

We should take the reggeization of the intermediate $\phi$ meson into account. We consider two prescriptions of the reggeization (only expected to hold in the $\left|\hat{p}^{2}\right| / s_{34} \ll 1$ regime):

$\Delta_{\mu \nu}^{(\phi)}(\hat{p}) \rightarrow \Delta_{\mu \nu}^{(\phi)}(\hat{p})\left(\exp \left(i \phi\left(s_{34}\right)\right) \frac{s_{34}}{s_{\mathrm{thr}}}\right)^{\alpha_{\phi}\left(\hat{p}^{2}\right)-1}$

ISSN 2071-0194. Ukr. J. Phys. 2019. Vol. 64, No. 8 
$\phi\left(s_{34}\right)=\frac{\pi}{2} \exp \left(\frac{s_{\mathrm{thr}}-s_{34}}{s_{\mathrm{thr}}}\right)-\frac{\pi}{2}$,

where $s_{\mathrm{thr}}=4 m_{\phi}^{2}$. Alternatively, we use

$$
\begin{aligned}
& \Delta_{\rho_{1} \rho_{2}}^{(\phi)}(\hat{p}) \rightarrow \Delta_{\rho_{1} \rho_{2}}^{(\phi)}(\hat{p}) F\left(\mathrm{Y}_{\mathrm{diff}}\right)+ \\
& +\Delta_{\rho_{1} \rho_{2}}^{(\phi)}(\hat{p})\left[1-F\left(\mathrm{Y}_{\mathrm{diff}}\right)\right] \times \\
& \times\left(\exp \left(i \phi\left(s_{34}\right)\right) \frac{s_{34}}{s_{\mathrm{thr}}}\right)^{\alpha_{\phi}\left(\hat{p}^{2}\right)-1},
\end{aligned}
$$

where $F\left(\mathrm{Y}_{\text {diff }}\right)=\exp \left(-c_{\mathrm{y}}\left|\mathrm{Y}_{\text {diff }}\right|\right)$. Here, $c_{\mathrm{y}}$ is an unknown parameter which measures, how rapidly one approaches the Regge regime. This gives the proper Regge behavior for $s_{34}-4 m_{\phi}^{2} \gg 1 \mathrm{GeV}^{2}$; whereas, for smaller $s_{34}$, we have the mesonic behavior. We take $\alpha_{\phi}\left(\hat{p}^{2}\right)=\alpha_{\phi}(0)+\alpha_{\phi}^{\prime} \hat{p}^{2}, \alpha_{\phi}(0)=0.1$ [31], and $\alpha_{\phi}^{\prime}=0.9 \mathrm{GeV}^{-2}$.

In order to give realistic predictions, we shall include the absorption effects calculated at the amplitude level and related to the $p p$ nonperturbative interactions. The full amplitude includes the $p p$ rescattering corrections (absorption effects)

$$
\begin{aligned}
& \mathcal{M}_{p p \rightarrow p p \phi \phi}=\mathcal{M}^{\text {Born }}+\mathcal{M}^{\text {absorption }}, \\
& \mathcal{M}^{\text {absorption }}\left(s, \boldsymbol{p}_{1 t}, \boldsymbol{p}_{2 t}\right)= \\
& =\frac{i}{8 \pi^{2} s} \int d^{2} \boldsymbol{k}_{t} \mathcal{M}^{\text {Born }}\left(s, \tilde{\boldsymbol{p}}_{1 t}, \tilde{\boldsymbol{p}}_{2 t}\right) \mathcal{M}_{\mathrm{el}}^{(\mathbb{P})}\left(s,-\boldsymbol{k}_{t}^{2}\right),
\end{aligned}
$$

where $\tilde{\boldsymbol{p}}_{1 t}=\boldsymbol{p}_{1 t}-\boldsymbol{k}_{t}$ and $\tilde{\boldsymbol{p}}_{2 t}=\boldsymbol{p}_{2 t}+\boldsymbol{k}_{t} \cdot \mathcal{M}_{\mathrm{el}}^{(\mathbb{P})}$ is the elastic $p p$-scattering amplitude with the momentum transfer $t=-\boldsymbol{k}_{t}^{2}$.

\section{Results}

It is very difficult to describe the WA102 data for the $p p \rightarrow p p \phi \phi$ reaction including resonances and the $\phi$ exchange mechanism only [20]. Inclusion of the odderon exchange improves the description of the WA102 data [25]. The result of our analysis is shown in Fig. 2.

Having fixed the parameters of our quasifit to the WA102 data, we wish to show our predictions for the LHC. In Fig. 3, we show the results for the ATLAS experimental conditions $\left(\left|\eta_{K}\right|<2.5, p_{t, K}>\right.$ $>0.2 \mathrm{GeV})$. The distribution in the four-kaon invariant mass is shown in the top panel, and the difference in rapidities between the two $\phi$ mesons in the bottom panel. The small intercept of the $\phi$-reggeon exchange, $\alpha_{\phi}(0)=0.1$, makes the $\phi$-exchange contribution steeply falling with increasing $\mathrm{M}_{4 K}$ and $\left|\mathrm{Y}_{\text {diff }}\right|$. Therefore, an odderon with an intercept $\alpha_{\mathbb{O}}(0)$ around 1.0 should be clearly visible in the region of large four-kaon invariant masses and for large rapidity distance between the $\phi$ mesons.

\section{Conclusions}

By confronting our model results, including the odderon, the reggeized $\phi$ exchange, and the $f_{2}(2340)$ resonance exchange contributions, with the WA102 data from [25], we derived an upper limit for the $\mathbb{P}(\phi)$ coupling. advantage of this process for experimental studies is the following. With regard for the typical kinematic cuts for LHC experiments in the $p p \rightarrow p p \phi \phi \rightarrow p p K^{+} K^{-} K^{+} K^{-}$reaction, we have found that the odderon exchange contribution should be distinguishable from other contributions for a large rapidity distance between the outgoing $\phi$ mesons and in the region of large four-kaon invariant masses. At least, it should be possible to derive an upper limit on the odderon contribution in this reaction.

Our results can be summarized in the following way:

- CEP is a particularly interesting class of processes which provides insight to the unexplored soft QCD phenomena. The fully differential studies of the exclusive $p p \rightarrow p p \phi \phi$ reaction within the tensorpomeron and vector-odderon approaches were executed; for more details, see [20].

- Integrated cross-sections of order of a few nb are obtained, including the experimental cuts relevant for the LHC experiments. The distribution in the rapidity difference of both $\phi$-mesons could shed light on the $f_{2}(2340) \rightarrow \phi \phi$ coupling, not known at present. Here, we used only one type of $\mathbb{P P} f_{2}$ coupling (out of 7 possible; see [14]). We have checked that, for the distributions studied here, the choice of $\mathbb{P P} f_{2}$ coupling is not important. This is a different situation compared to the one observed by us for the $p p \rightarrow p p\left(\mathbb{P P} \rightarrow f_{2}(1270) \rightarrow \pi^{+} \pi^{-}\right)$reaction [14].

- From our model, we have found that the odderonexchange contribution should be distinguishable from other contributions for a relatively large rapidity separation between the $\phi$ mesons.

Hence, to study this type of mechanism, one should investigate events with rather large four-kaon invariant masses, outside of the region of resonances. These 
events are then "three-gap events": proton-gap- $\phi$ gap- $\phi$-gap-proton. Experimentally, this should be a clear signature.

- Clearly, an experimental study of CEP of a $\phi$ meson pair should be very valuable for clarifying the status of the odderon. At least, it should be possible to derive an upper limit on the odderon contribution to this reaction.

I thank the Organizers of the New Trends in HighEnergy Physics conference in Odessa, Ukraine for the hospitality and good organization. P.L. was partially supported by the Polish Scientific Center of the PAS in Kiev.

1. T. Aaltonen et al. (CDF Collaboration). Measurement of central exclusive $\pi^{+} \pi^{-}$production in $p p$ collisions at $\sqrt{s}=$ $=0.9$ and $1.96 \mathrm{TeV}$ at CDF. Phys. Rev. D 91, 091101 (2015).

2. CMS Collaboration. Measurement of total and differential cross sections of central exclusive $\pi^{+} \pi^{-}$production in proton-proton collisions at 5.02 and $13 \mathrm{TeV}$. CMS-PASFSQ-16-006.

3. R. Sikora (STAR Collaboration). Recent results on Central Exclusive Production with the STAR detector at RHIC. arXiv:1811.03315 [hep-ex].

4. R. Staszewski, P. Lebiedowicz, M. Trzebinski, J. Chwastowski, A. Szczurek. Exclusive $\pi^{+} \pi^{-}$production at the LHC with forward proton tagging. Acta Phys. Polon. B 42, 1861 (2011).

5. L. Łukaszuk, B. Nicolescu. A possible interpretation of pp rising total cross-sections. Lett. Nuovo Cim. 8, 405 (1973).

6. A. Breakstone. Measurement of $\bar{p} p$ and $p p$ elastic scattering in the dip region at $\sqrt{s}=53 \mathrm{GeV}$. Phys. Rev. Lett. 54, 2180 (1985).

7. TOTEM Collaboration. First determination of the $\rho$ parameter at $\sqrt{s}=13 \mathrm{TeV}$ - probing the existence of a colourless three-gluon bound state. arXiv:1812.04732 [hepex]; arXiv:1812.08610 [hep-ex].

8. A. Schäfer, L. Mankiewicz, O. Nachtmann. Doublediffractive $J / \psi$ and $\varphi$ production as a probe for the odderon. Phys. Lett. B 272, 419 (1991).

9. C. Ewerz. The odderon in quantum chromodynamics. arXiv:0306137 [hep-ph].

10. L. Adamczyk et al. (STAR Collaboration). Single spin asymmetry AN in polarized proton-proton elastic scattering at $\sqrt{s}=200 \mathrm{GeV}$. Phys. Lett. B 719, 62 (2013).

11. A. Bolz, C. Ewerz, M. Maniatis, O. Nachtmann, M. Sauter, A. Schoning. Photoproduction of $\pi^{+} \pi^{-}$pairs in a model with tensor-pomeron and vector-odderon exchange. JHEP 1501, 151 (2015).

12. P. Lebiedowicz, O. Nachtmann, A. Szczurek. Exclusive central diffractive production of scalar and pseudoscalar mesons; tensorial vs. vectorial pomeron. Annals Phys. 344, 301 (2014).

13. P. Lebiedowicz, O. Nachtmann, A. Szczurek. $\rho^{0}$ and DrellSoding contributions to central exclusive production of $\pi^{+} \pi^{-}$pairs in proton-proton collisions at high energies. Phys. Rev. D 91, 074023 (2015).

14. P. Lebiedowicz, O. Nachtmann, A. Szczurek. Central exclusive diffractive production of the $\pi^{+} \pi^{-}$continuum, scalar, and tensor resonances in $p p$ and $p \bar{p}$ scattering within the tensor Pomeron approach. Phys. Rev. D 93, 054015 (2016).

15. P. Lebiedowicz, O. Nachtmann, A. Szczurek. Exclusive diffractive production of $\pi^{+} \pi^{-} \pi^{+} \pi^{-}$via the intermediate $\sigma \sigma$ and $\rho \rho$ states in proton-proton collisions within tensor Pomeron approach. Phys. Rev. D 94, 034017 (2016).

16. P. Lebiedowicz, O. Nachtmann, A. Szczurek. Central production of $\rho^{0}$ in $p p$ collisions with single proton diffractive dissociation at the LHC. Phys. Rev. D 95, 034036 (2017).

17. P. Lebiedowicz, O. Nachtmann, A. Szczurek. Central exclusive diffractive production of $p \bar{p}$ pairs in proton-proton collisions at high energies. Phys. Rev. D 97, 094027 (2018).

18. P. Lebiedowicz, O. Nachtmann, A. Szczurek. Towards a complete study of central exclusive production of $\mathrm{K}^{+} \mathrm{K}^{-}$ pairs in proton-proton collisions within the tensor Pomeron approach. Phys. Phys. D 98, 014001 (2018).

19. P. Lebiedowicz, O. Nachtmann, A. Szczurek. Extracting the pomeron-pomeron- $f_{2}(1270)$ coupling in the $p p \rightarrow$ $\rightarrow p p \pi^{+} \pi^{-}$reaction through angular distributions of the pions. arXiv:1901.07788 [hep-ph].

20. P. Lebiedowicz, O. Nachtmann, A. Szczurek. Central exclusive diffractive production of $K^{+} K^{-} K^{+} K^{-}$via the intermediate $\phi \phi$ state in proton-proton collisions. Phys. Rev. D 99, 094034 (2019).

21. C. Ewerz, M. Maniatis, O. Nachtmann. A model for soft high-energy scattering: Tensor pomeron and vector odderon. Annals Phys. 342, 31 (2014).

22. C. Ewerz, P. Lebiedowicz, O. Nachtmann, A. Szczurek. Helicity in proton-proton elastic scattering and the spin structure of the pomeron. Phys. Lett. B 763, 382 (2016).

23. A. Etkin et al. Increased statistics and observation of the $g_{T}, g_{T^{\prime}}$, and $g_{T^{\prime \prime}} 2^{++}$resonances in the Glueball enhanced channel $\pi^{-} p \rightarrow \varphi \varphi n$. Phys. Lett. B 201, 568 (1988).

24. D. Aston et al. Strangeonium production from LASS. Nucl. Phys. Proc. Suppl. 21, 5 (1991).

25. D. Barberis et al. (WA102 Collaboration). A Study of the centrally produced phi phi system in $p p$ interactions at 450-GeV/c. Phys. Lett. B 432, 436 (1998).

26. M. Ablikim et al. (BESIII Collaboration). Observation of pseudoscalar and tensor resonances in $J / \psi \rightarrow \gamma \phi \phi$. Phys. Rev. D 93, 112011 (2016).

27. C.J. Morningstar, M.J. Peardon. Glueball spectrum from an anisotropic lattice study. Phys. Rev. D 60, 034509 (1999).

28. R. Fiore, L. Jenkovszky, R. Schicker. Resonance production in Pomeron-Pomeron collisions at the LHC. Eur. Phys. J. $C$ 76, 38 (2016).

ISSN 2071-0194. Ukr. J. Phys. 2019. Vol. 64, No. 8 
29. R. Fiore, L. Jenkovszky, R. Schicker. Exclusive diffractive resonance production in proton-proton collisions at high energies. Eur. Phys. J. C 78, 468 (2018).

30. A. Donnachie, H.G. Dosch, P.V. Landshoff, O. Nachtmann. Pomeron Physics and QCD (Cambridge Univ. Press, 2002).

31. P.D.B. Collins. Regge Theory and Particle Physics (Univ. of Durham, 1970).

Received 08.07.19

П. Лебєдович

ПОШУКИ ОДДЕРОНА

В ЕКСКЛЮЗИВНИХ РЕАКЦІЯХ

$\mathrm{P}$ е $з$ ю м е

Обговорюємо можливість користуватися процесом $p p \rightarrow$ $\rightarrow p p \phi \phi$ для ідентифікації обміну оддероном. До цього часу немає однозначного експериментального доказу існування оддерона - партнера померона з негативним зарядовим спряженням, $C=-1$. Минулорічні результати Колаборації ТОТЕМ вказують на те, що оддерон може спричиняти розбіжність між теоретичними розрахунками та даними ТОТЕМ про пружне розсіяння протонів. Ми презентуємо нові результати досліджень центрального ексклюзивного народження (CEP) пар $\phi \phi$ в протонних зіткненнях. Ми разглядаємо фузію померонів у $\phi \phi(\mathbb{P P} \rightarrow \phi \phi)$ через континуум завдяки обміну в $\hat{t}$ - і $\hat{u}$-каналах реджезованого $\phi$ мезона, фотона та оддерона, а також резонансного процесу в $s$-каналі $\left(\mathbb{P P} \rightarrow f_{2}(2340) \rightarrow \phi \phi\right)$. Частинка $f_{2}$ є кандидатом на тензорний глюбол. Амплітуда процесу формулюється в рамках підходу, де померон є тензором, а оддерон є вектором. Деякі з параметрів моделі визначаються з порівняння 3 експериментальними даними WA102. Дані WA102 не виключають обмін оддероном для великих інваріантних мас $\phi \phi$. Сигнал з великими значеннями $M_{\phi \phi}$ або $Y_{\text {diff }}$ на LHC буде таким чином вказувати на присутність обміну оддероном. Цей процес привабливий ще тим, що в ньому оддерон не прив'язується до протона. 